\title{
MANET of an Effective Defence Scheme for Suggestion Based Trust Model
}

\author{
Barath Kumar R, Stalin Alex
}

\begin{abstract}
The immovable nature of passing on packages through multi -bounce middle hubs is a critical problem in the versatile impromptu organizations (MANETs). The disseminated versatile hubs set up associations with structure the MANET, which may incorporate childish and getting into mischief hubs. Suggestion based trust the board is proposed in the creating as a system to evaluate through the acting up hubs while looking for a bundle conveyance course. Nonetheless, building a trust model that embraces suggestions by different hubs in the organization is a difficult issue because of the danger of deceptive proposals like reviling, voting form stuffing, and conspiracy. we examines the issues identified with assaults presented by getting rowdy hubs while proliferating suggestions in the current trust models. We propose a suggestion based trust model with a safeguard plot, which uses grouping method to progressively sift through assaults identified with exploitative proposals between certain time dependent on number of collaborations, similarity of data and closeness between the hubs. We evaluate the trust degree as two cases like direct and indirect trust values between neighboring nodes from source. To form a clustering routing network from similar trust values from $S$ to $D$.The model is experimentally tried under a few portable and detached geographies in which hubs experience changes in their local prompting regular course changes. The observational investigation shows heartiness and exactness of the trust model in a dynamic MANET climate.

Keywords: Dynamically Filter, Clustering, AODV Protocol,
\end{abstract} MANET

\section{INTRODUCTION} Wireless sensor Network (WSN) organization and actuator networks are spatially dispersed self-sufficient sensor to screen physical or climate conditions, for example, temperature, sound, pressure and so on and to helpfully go their information through the organization to different areas. A WSN framework joins an entryway that gives remote network back to the wired world and circulated hubs.A versatile specially appointed organization (MANET), otherwise called remote impromptu organization or specially appointed remote organization is a consistently self-designing, framework less organization of cell phones associated remotely.

Manuscript received on June 02, 2021

Revised Manuscript received on June 09, 2021.

Manuscript published on June 30, 2021.

* Correspondence Author

Barath Kumar R*, M.Tech, Department of Computer Science and Engineering, SET-Jain University, Bengaluru (Karnataka), India.

Dr. Stalin Alex, Assistant Professor, Department of Computer Science and Engineering, SET-Jain University, Bengaluru (Karnataka), India.

(C) The Authors. Published by Blue Eyes Intelligence Engineering and Sciences Publication (BEIESP). This is an open access article under the CC BY-NC-ND license (http://creativecommons.org/licenses/by-nc-nd/4.0/)
Remote Sensor Networks, here and there called

Overseeing trust in a dispersed versatile specially appointed organization (MANET) is a difficult when coordinated effort or participation is basic to accomplishing mission and framework objectives, for example, dependability, accessibility, adaptability, and reconfigurability. In characterizing and overseeing trust in a military MANET, we should consider the cooperations between the composite psychological, social data and correspondence organizations, and consider the extreme asset imperatives (e.g., registering power, energy, transmission capacity, time), and elements (eg., topology changes, hub portability, hub disappointment, spread channel conditions). In ref. [1], the creator proposed a Stream Position Performance Analysis (SPPA) approach. This methodology screens the situation of any field station in sending the data to play out a Distributed Denial of Service (DDoS) assault. The strategy registers different elements like Conflict field, Conflict information and Attack signature test rate (CCA). Utilizing every one of these variables, the strategy distinguishes the reliability of the parcel and remembers it for dynamic. The proposed approach builds the exhibition of a Distributed Denial of Service (DDoS) assault location in a VANET climate. In ref. [2], the creator Vehicular Ad Hoc Network (VANET) has charmed immense examination, to update street wellbeing and diversion for the explorer, by bestowing constant data among vehicles.In ref. [3], the creator presents a fluffy rationale based multi-models insightful forward directing convention. Which is operable in powerful organization condition and viable with both correspondence modes like vehicle-to-vehicle and vehicle-to-foundation. To choose the insatiable next hub for information sending our proposed convention compute hub security cost and positive advancement esteem.In ref. [4],the idea of vehicle grouping is acquainted in VANET with improve network execution by taking care of every such issue. This paper gives an inside and out order of grouping conventions in VANET dependent on their plan targets.

In ref. [5],the creator centers around the solidified methodology named, IDS and Trust arrangement Collaborated with Acknowledge based methodology (ITCA), performs distinguishing proof of assault, detachment of acting mischievously hubs and control conduct of hubs in the organization.In ref. [6]A dynamic trust forecast model to assess the reliability of hubs, which depends on the hubs' authentic practices, just as the future practices through broadened fluffy rationale rules expectation.

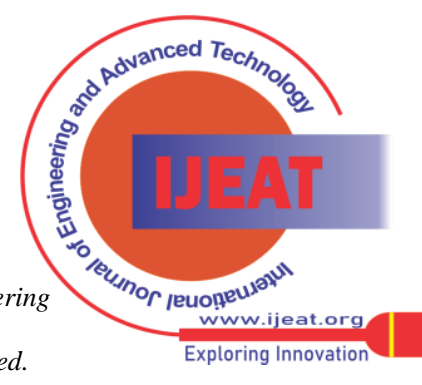


The constraint of this paper is issue of dynamic conduct alteration isn't thought of. In ref. [7]Ad hoc arrangements, because of their ad libbed nature, are as often as possible set up in shaky conditions and subsequently become powerless to assaults.In ref. [8] The security related issues and difficulties in WSNs are examined. We recognize the security dangers and survey proposed security systems for WSNs. The restriction of this paper is cryptography, key administration, secure steering, secure information conglomeration and interruption recognition in WSNs, there are still a few difficulties to be tended to. Some security issues including security-energy appraisal, information confirmation, survivability, trust, start to finish security, security and protection uphold for datacentric sensor organizations (DCS) and hub bargain circulation are should have been finished.

\section{PROBLEM DEFINITION}

The existing approach using dynamic source routing (DSR) protocol to identify the misbehaving nodes. Utilizing bunching strategy to progressively sift through assaults identified with unscrupulous proposals between specific occasions dependent on number of communications, similarity of data and closeness between the hubs. The first builds up trust connections between hubs dependent on direct collaborations as it were. The subsequent kind depends on direct perceptions of the hub itself and suggestions gave by other by different hubs in the organization. The utilization of suggestion based trust strategy can be favorable to hubs in finding getting out of hand hubs.Dynamic determination of the quantity of suggestions dependent on a timeframe can have numerous points of interest,

- Decreasing intricacy and Space utilization

- Reject an old suggestion from the count

- Decreasethe time that is utilized to choose reliable group.

\section{AODVARRANGEMENTS}

Ad hoc On-Demand Distance Vector (AODV) Steering is a handlingprotocol for versatile unprepared organizations (MANETs) and other remote specially appointed organizations. AODV, the organization is calm until an affiliation is required. Then the association center that needs an affiliation conveys a requesting for affiliation. Other AODV centers forward particular message, and recording the center that they heard it from, making an impact of fleeting courses back to the down and out center point. Exactly when a center gets such a message and has a course to the ideal center, it conveys something explicit in opposite through an ephemeral course to the referencing center. The helpless center by then beginnings using the course that has the most un-number of bobs through various centers. Unused segments in the controlling tables are reused after a period. Exactly when an association misses the mark, a coordinating misstep is passed back to a sending center point, and the cycle goes over. The potential gain of AODV is that it makes no extra traffic for correspondence along existing associations. In like manner, distance vector coordinating is direct, and needn't bother with a great deal of memory or figuring. In any case AODV requires greater chance to develop an affiliation, and the hidden correspondence to set up a course is heavier than some various techniques.

\section{SYSTEM MODEL}

Here we consider a WSN comprising of a couple of sink hubs and various sensor hubs that are haphazardly dispersed in an assigned region. Every sensor hub is responsible for both identifying occasions and going about as a switch to advance parcels. All the sensor hubs are asset compelled and have a similar restricted radio inclusion. Therefore, start to finish correspondence in a WSN is typically accomplished through multi-jump transferring where a correspondence way is set up in a disseminated way.

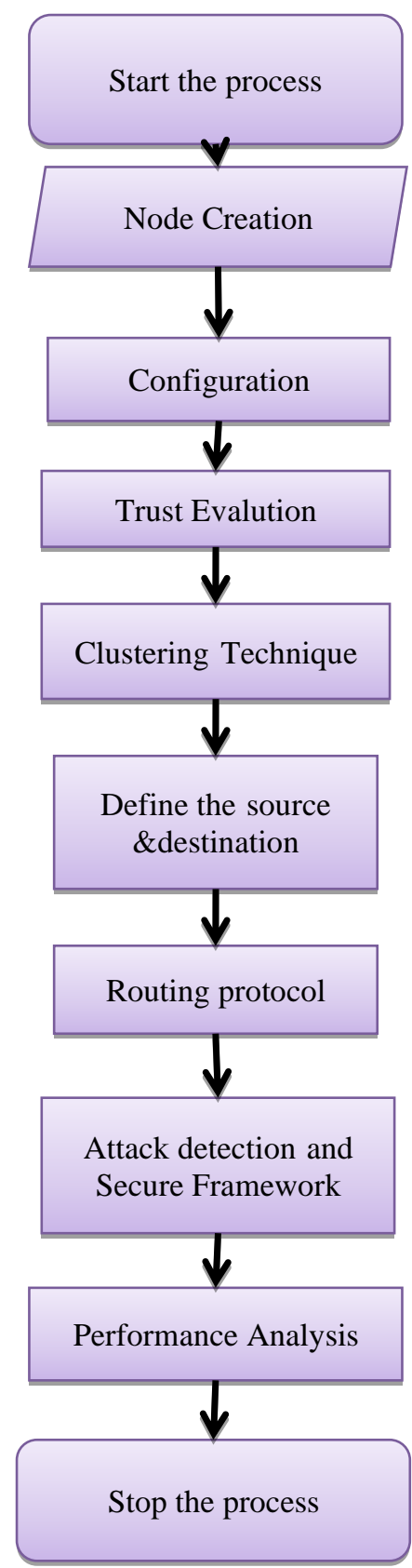

Fig. 1. Flow diagram of proposed.

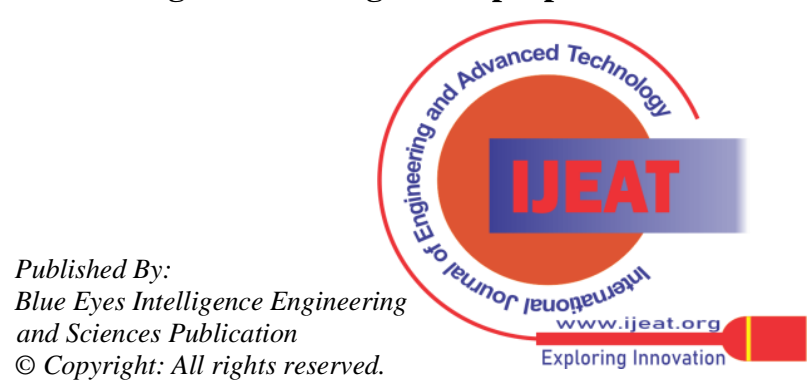


Model of trust basically performs the determination of trust, Computation, and application. In this paper, we receive guard $\mathrm{dog}$ as the establishment of discovery instruments. Every sensor hub is liable for observing the conduct of its. Neighbors and assessing their trust level. All the more explicitly, the identification results are used for the proof of trust calculation. $(i, j)$ speaks to the trust estimation of hub $j$ for hub $i$. In our model, hub $i$ is the assessing gadget and hub $j$ is the assessed one. The trust tof a subjective hub incorporates direct trust $d t$ and backhanded trust $i t$.

$>$ Direct trust depends on direct perceptions of every hub that takes an interest in information correspondence.

$>$ Indirect trust which is additionally called suggestion trust, represents the trust relations between disseminated hubs without direct associations.

\subsection{Trust Nodes}

Typically, the sensor hubs are exceptionally obliged regarding computational force, energy, memory, and transfer speed, so the plan of security components for WSNs is essentially testing.In addition, the trust value is subject to 0 $\leq t \leq 1$.

\subsection{Trust Paths}

When the point of hub source gets ready to send bundles to receiver hub by means of multi-hop correspondence, it should assess the trust estimation of the course. Various techniques dependent on the consequences of hubs' trust evaluation can be applied to the cycle of way trust calculation.

\subsection{Routing Scheme}

$>$ When the point of hub source plans to sendbundles to receiverhub, hub introduces the trust inference measure and sends a trust demand parcel to its neighbor hubs. The trust demand parcel comprises of assessing hub's ID and is the assessed hubs' ID. To decrease the overhead of trust induction system, as far as possible estimation of the trust demand parcel ought to be set to one. This worth ought to be decremented by one each time the trust demand bundle is sent in the event that it isn't zero. The hub that gets the trust demand parcel should initially check on the off chance that it has just gotten a similar solicitation. On the off chance that it has, the solicitation ought to be quickly disposed of. If not, the hub should communicate this trust solicitation to every one of its neighbors.

$>$ When accepting the trust demand parcel, the hubs aside from the assessing one should check whether the assessed hub is its neighbor (the source hub will basically dispose of this solicitation as it is the wellspring of the trust demand). If not, it stays quiet. Something else, the hubs may unicast a trust answer to the assessing hub (the source hub) through the current converse courses. At that point, these hubs will drop the transmission trust demand bundles if as far as possible

$>$ After acquiring the proposals gave by the neighbors of the assessed hub, the assessing hub figures the trust an incentive by consolidating direct trust with circuitous trust. At that point, hub can decide if the assessed hub worth is equivalent to zero.

ought to be trusted by the necessary way trust imperative. Likewise, hub can locate a confided in sending set and send the course solicitation to these hubs in the sending set.

$>$ If a moderate confided in hub that gets the course demand has the ideal course to the objective hub, it will send a course answer to the source hub. At that point, the source hub can locate the ideal course to the objective hub.

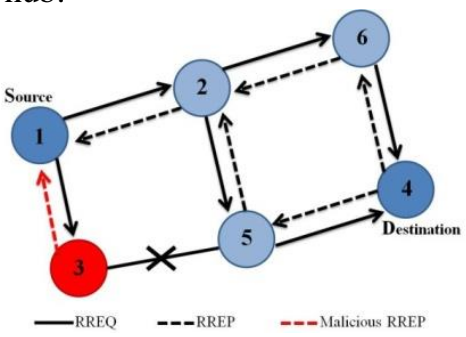

Fig. 2. Routing Algorithm.

$>$ Once the course demand hits the objective, the objective hub will send a course answer to the source hub by means of the chose turn around course. At last, hub can send information parcels to hub through the ideal course.

$>$ When the transmission of parcels from source to objective by means of the confided in arrangement of hubs, if a gatecrasher is wrongly remembered for the confided in arrangement of hubs, it very well may be effortlessly recognized as it carries on contrastingly to various hubs. Here the malignant conduct can be distinguished by the neighbors. At that point an other believed course is resolved again as referenced in the past advances and the bundles are sent.

\section{SIMULATION RESULTS}

Network Simulator (NS) is a name of arrangementsin a freestanding occasion examination of network systems, explicitly NS-1, NS-2 and NS-3. Each and every one of them are freestanding-occasion examination of network system, Generally apply in examination and instructing. Mainly NS-2 for Network Simulator.

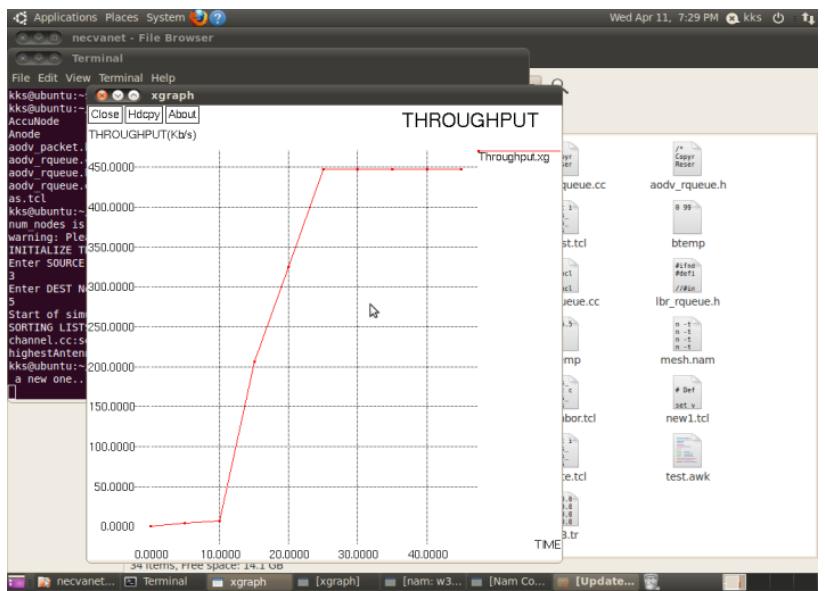

Fig. 3 Network Simulation for Throughput

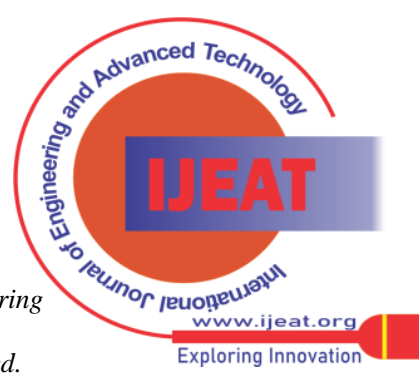




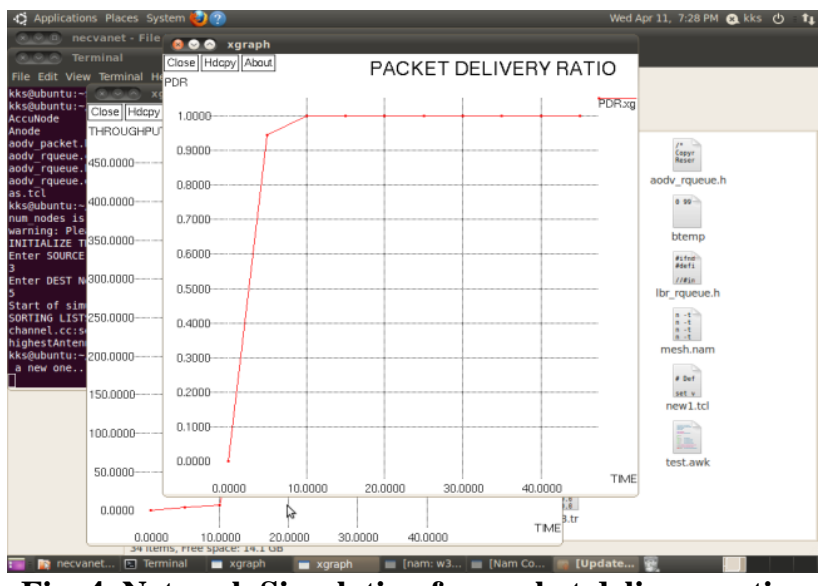

Fig. 4. Network Simulation for packet delivery ratio.

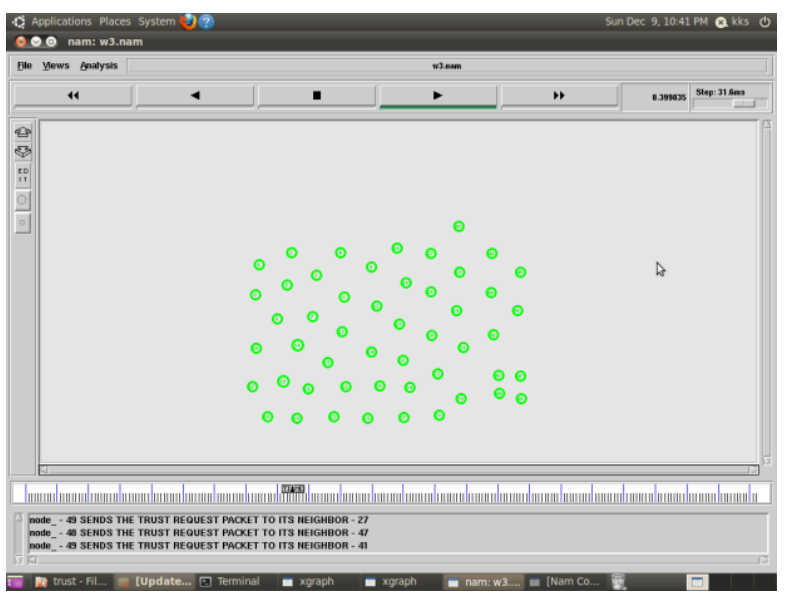

Fig. 5. Number of nodes.

\section{CONCLUSION}

Suggestion based trust model with a guard plan to sift through assaults identified with deceptive proposals. The suggesting hub is picked dependent up on 3 elements to controlits genuineness number of associations with the assessed hub, solidarity point of view with the assessing hub for tackling the issue of the shortage of information, nearly to the assessing hub.

\section{REFERENCES}

1. R. Kolandaisamy, R. M. Noor, I. Kolandaisamy, I. Ahmedy, M.L.M. Kiah, M.E.M. Tamil andT. Nandy,"A stream position performance analysis model based on DDoS attack detection for cluster-based routing in VANET", Journal of Ambient Intelligence and Humanized Computing, pp. 1-14, 2020.

2. A. Srivastava, A. Prakash, and R.Tripathi, "Location based routing protocols in VANET: Issues and existing solutions", Vehicular Communications, vol. 23, 100231, 2020.

3. R. Purkait, andS. Tripathi, "Fuzzy logic based multi-criteria intelligent forward routing in VANET", Wireless Personal Communications, vol. 111 no. 3, pp. 1871-1897, 2020.

4. A. Katiyar, D. Singh, andR. S. Yadav, "State-of-the-art approach to clustering protocols in vanet: a survey", Wireless Networks, vol. 26 no. 7, pp. 5307-5336.

5. N. Marathe, and S. K.Shinde, "ITCA, an IDS and trust solution collaborated with ACK based approach to mitigate network layer attack on MANET routing", Wireless Personal Communications, vol. 107, no. 1, pp. 393-416 2020

6. KannanGovindan, Member IEEE and PrasantMohapatra, Fellow IEEE, "Trust Computations and Trust Dynamics in Mobile AdhocNetworks:A Survey", vol 14, no.2,Second Quarter 2012.

7. .PrayagNarula, Sanjay Kumar Dhurandher, SudipMisra, Isaac Woungang, "Security in mobile ad-hoc networks using soft encryptionand trust-based multi-path routing"vol. 3, no. 3, pp. $257-$ 279, May 2010.

Published By:

Blue Eyes Intelligence Engineering and Sciences Publication
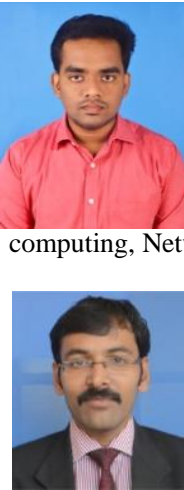
Networks: A survey”vol.18, no.2,Second Quarter 2016.

9. FarrukhAslam Khan, Muhammad Imran, Haider Abbas, Muhammad HanifDurad, "A detection and prevention system against collaborative attacks in mobile ad hoc networks" vol. 7, no. 1, February 2016

10. SilvereMavoungou, Georges Kaddoum, MostafaTaha, "Survey on threats and attacks on mobile networks", July 2016

\section{AUTHORS PROFILE}

Barath Kumar, received B. Tech degree in Computer Science Engineering from Vinayaka Missions University, Salem, Tamilnadu, India in the year 2016,currently pursuing his Final year M.tech in Cyber Security from Jain University, Bengaluru, Karnataka. Hardware and Networking Analyst - Certified by DOTE, Chennai. His areas of interest are in the field of, cloud computing, Network Security, internet of things,and automation.

Dr. D. Stalin Alex, M.I.S.T.E. M.Tech. Ph.D, Associate Professor \& Program In charge Department of Computer Science \& Engineering (Data Science) School of Engineering \& Technology, Jain University, JGI Global Campus, Jakkasandra Post Kanakapura Taluk Ramanagara District, Bangalore- 562112, INDIA. 